\title{
Acute colonic pseudo-obstruction in a patient following total abdominal hysterectomy and bilateral salpingo-oophorectomy (Ogilvie syndrome)
}

\author{
D S Weerasekera ${ }^{a}$, W D D de Silva ${ }^{b}$, N K Rodrigo ${ }^{c}$, T K I N Nanayakkara ${ }^{d}$
}

\begin{abstract}
A 50-year-old woman underwent total abdominal hysterectomy and bilateral salphingo-oophorectomy for heavy menstrual bleeding due to a uterine fibroid. On second post-operative day she developed progressive abdominal distention, tachycardia, and fever. An intra-abdominal pathology was suspected and she was re-opened on the third postoperative day. At laparotomy a distended colon was found with serosal tears due to distention with no distal colonic obstruction. Bowel decompression was done and a diagnosis of acute colonic pseudo-obstruction (ACPO) was made. Since her abdominal distention was persisting after the laparotomy she was treated with intravenous neostigmine. She responded immediately with passage of flatus and bowel opening. Subsequent recovery was uneventful. ACPO is a clinical entity characterized by severe colonic distention in the absence of mechanical obstruction. It can result in bowel ischaemia and perforation if left untreated. Therapeutic importance of neostigmine is discussed in the management of this potentially fatal condition.
\end{abstract}

Key words: acute colonic pseudo-obstruction, neostigmine, colonic distention, bowel perforation

Sri Lanka Journal of Obstetrics and Gynaecology 2020; 42: 157-160

DOI: http://doi.org/10.4038/sljog.v42i4.7970

a Professor of Obstetrics and Gynaecology, Department of Obstetrics and Gynaecology, Sir John Kotelawala Defence University, Sri Lanka.

b Senior Lecturer in Surgery, Department of Surgery, Sir John Kotelawala Defence University, Sri Lanka.

c Senior Lecturer in Obstetrics and Gynaecology, Sir John Kotelawala Defence University, Sri Lanka.

d Medical Officer, Department of Obstetrics and Gynaecology, University Hospital, Kotelawala Defence University, Sri Lanka.

Correspondence: e-mail: deepalsenaka1@gmail.com

(D) https://orcid.org//0000-0001-5857-5219

Received 29th September 2020

Accepted $20^{\text {th }}$ November 2020

This is an open-access article distributed under the terms of the Creative Commons Attribution 4.0 International License, which permits unrestricted use, distribution and reproduction in any medium provided the original author and source are credited. 


\section{Introduction}

Ogilvie's syndrome describes an acute colonic pseudoobstruction (ACPO) consisting of dilatation of part or all of the colon and rectum without intrinsic or extrinsic mechanical obstruction. It often occurs in debilitated patients. Its pathophysiology is still poorly understood ${ }^{1}$. The colon is often significantly dilated. Symptoms are similar to other forms of intestinal obstruction and can include nausea, vomiting, abdominal bloating or swelling and constipation. Ogilvie syndrome is usually associated with co-morbid conditions, such as severe musculoskeletal abnormalities, trauma, surgery, or sepsis. But here we describe a patient who developed acute colonic pseudo-obstruction following total abdominal hysterectomy with no co-morbid factors.

\section{Case report}

A 50 year old previously healthy woman, who is a mother of 3 children delivered vaginally presented to the clinic with a history of regular heavy menstrual bleeding not responding to medical management for the past one year duration with a haemoglobin of $9 \mathrm{~g} / \mathrm{dl}$. Ultrasound scan showed an enlarged uterus with a $6.4 \times 5.5 \times 5.0 \mathrm{~cm}$ size fibroid in the posterior myometrium. Total abdominal hysterectomy and bilateral salpingo-oophorectomy was planned after discussing the management options with the patient. Routine total abdominal hysterectomy and bilateral salpingooophorectomy was done and the surgery was uncomplicated.

Patient had a normal recovery by postoperative day one. By day two she was started on normal diet and mobilized, but by evening she developed gradual abdominal distention with non passage of flatus. Nevertheless, the bowel sounds were present. On examination she was afebrile, not pale with a pulse rate of $88 \mathrm{bpm}$, blood pressure 120/80mmHg. Patient was kept on clear fluids and analgesics were given for the pain relief. By the night of second post-operative day patient was complaining of worsening abdominal pain and distention. Pulse rate was gradually rising up to $120 \mathrm{bpm}$, with a blood pressure of 120/80. Naso-gastric tube was inserted and she was kept nil orally. She was started on intravenous co-amoxiclav and intravenous metronidazole. Erect X-ray abdomen was done and it showed dilated large bowel loops (Figure 1).

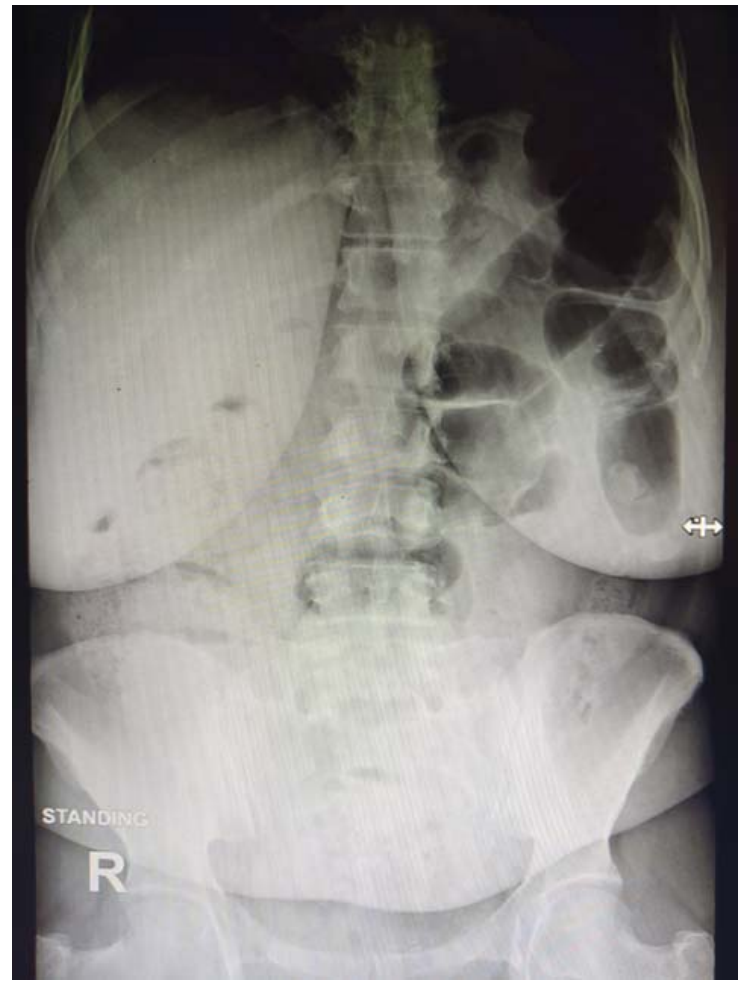

Figure 1. X-ray erect abdomen on day three of surgery.

By the early morning of day three patient developed fever spikes up to $101 \mathrm{~F}$ with worsening abdominal pain and absolute constipation. Pulse rate was rising up to $135 \mathrm{bpm}$ with blood pressure $120 / 80 \mathrm{mmHg}$. Respiratory rate was $32 / \mathrm{min}$. Per rectal examination revealed an empty rectum. Intravenous meropenem was started after taking blood for culture. Urgent USS was done and it did not show any collection of fluid apart from dilated large bowel loops. Full blood count showedWBC $19 \times 10^{3}$ with a neutrophil leukocytosis, normal hemoglobin and platelet count, C-reactive protein of 197, serum creatinine serum electrolytes and chest X-ray were normal.

Patient was reviewed with the surgical team and a decision was made to reopen the abdomen in view of the persistent abdominal distention, marked left iliac fossa tenderness and deteriorating clinical condition over the past 24 hours. Emergency laparotomy was performed with a midline incision. During the surgery it was noted that the large bowel loops were grossly distended from caecum to the rectum with a competent ileocaecal valve. The small bowel was normal. Three points of serosal tears noted on transverse colon occurring most likely due to the closed loop obstruction. There was no evidence of any large bowel or rectal masses, bowel injury or active bleeding. Faecal impaction in distal rectum was found. Peritoneal fluid 


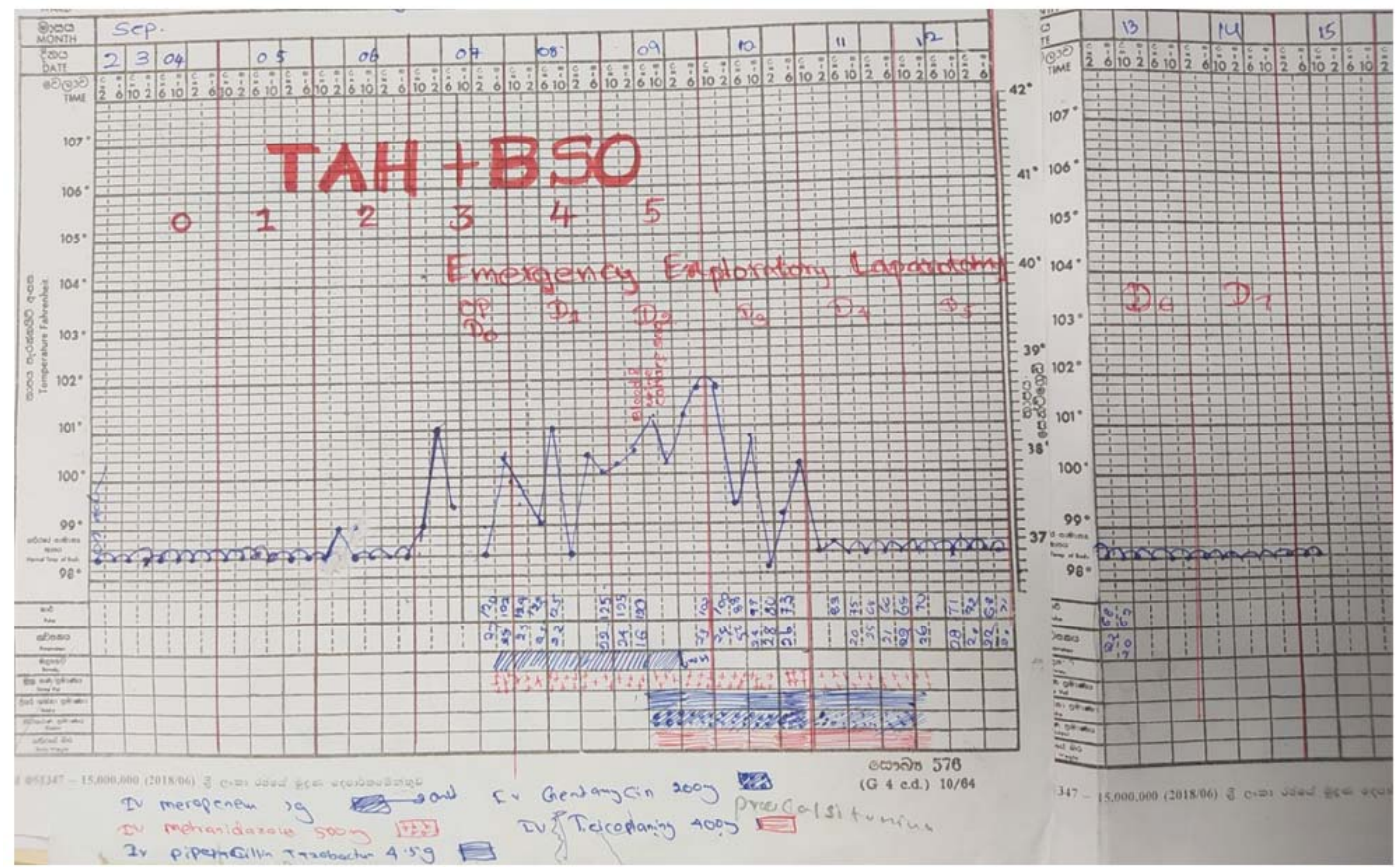

Figure 2. Temperature monitoring chart.

taken for culture and ABST, serosal tears were sutured, large bowel was decompressed, peritoneal washing done and incision closed after inserting a drain. A probable diagnosis of acute colonic pseudo-obstruction was made.

Patient was transferred to ICU for observation. Patient's intra abdominal pressure was monitored by connecting a manometer to the urinary catheter. Despite the decompression the patient developed abdominal distention again and was not clinically improving. Twenty-four hours after the laparotomy, tachycardia was persisting $>120$ bpm with ongoing fever spikes and intra abdominal pressure was gradually rising up to $16 \mathrm{mmHg}$. Full blood count showed WBC of $17 \times 10^{3}$ with neutrophil leukocytosis, CRP 284, serum electrolytes and creatinine were within normal range. Repeat X-ray abdomen showed dilated large bowel loops.

Intravenous meropenem was changed to intravenous piperacillintazobactam, teicoplaninand and gentamicin. Since the colonic distention was persisting a decision was made to treat her with intravenous neostigmine. Three $2 \mathrm{mg}$ doses were given 5 hours apart. Within few minutes of the first dose of neostigmine her bowels open and the distention was relieved. By the next day patient improved dramatically. Fever spikes were coming down, pulse rate reduced to $80-90 \mathrm{bpm}$, respiratory rate reduced to 20/min, intra abdominal pressures was reducing. By the fourth post operative day patient was afebrile and abdomen was not distended with regular bowel openings. Patient was transferred back to ward and discharged after a good recovery on day 7 of laparotomy.

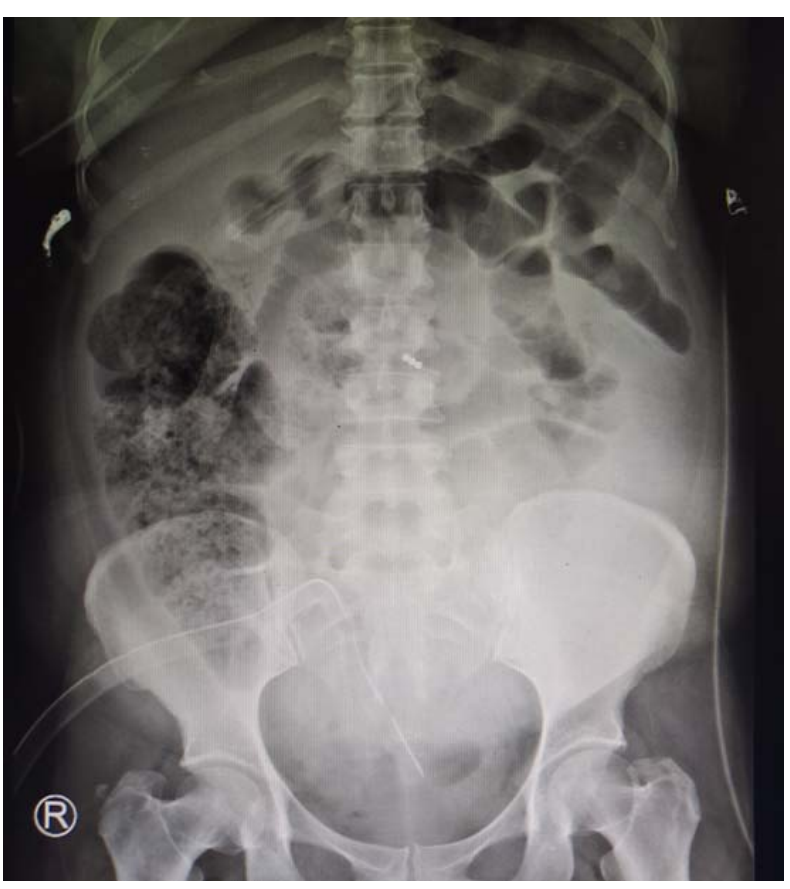

Figure 3. Repeated erect abdominal $\mathrm{X}$ ray on day two after re-opening. 


\section{Discussion}

Acute colonic pseudo-obstruction (ACPO) is a clinical entity characterized by severe colonic distention in the absence of mechanical obstruction ${ }^{2}$. The exact pathogenesis of this condition is not resolved yet but it is thought to result from an imbalance of the autonomic nervous system as a result of acute illness within the background of chronic disease. Often this is typically described in elderly patients and those suffering from multiple co-morbidities and after nonabdominal surgery ${ }^{3}$. It is extremely rare for it to occur after uncomplicated abdominal hysterectomy like in this patient with no comorbidities. A high clinical suspicion is extremely important for early diagnosis as massive colonic dilatation can cause ischaemia and perforation of the colon. ACPO has also been reported following caesarean section, instrumental delivery and even normal delivery ${ }^{2}$.

Diagnosis of ACPO is one of exclusion. It is essential to rule out any mechanical obstruction. Plain X-ray abdomen will show features of proximal colonic dilatation. As long as the patient is stable, has no peritoneal signs, and the caecal diameter is less than $12 \mathrm{cms}$ on radiographic imaging, conservative management can be continued. Conservative measures involve correcting electrolyte abnormalities, discontinuing opiates, nasogastric tube decompression, correcting any fluid imbalance and discontinuing oral intake ${ }^{4}$. All these measures were done on this patient but since there was not much improvement the decision was made to re-open. Already splitting of the colonic serosa was noted in several places at laparotomy.

When the colonic dilatation is persisting current recommendation is to proceed with intravenous neostigmine with cardiac monitoring ${ }^{5}$. Neostigmine is a parasympathomimetic, which acts by inhibiting acetylcholinesterase. This allows for increased acetylcholine availability and direct parasympathetic stimulation by nicotinic and muscarinic receptors ${ }^{6}$. Administration of neostigmine was associated with immediate peristalsis of the colon with the patient passing flatus and opening of bowel. Parasympathetic stimulation may cause profound bradycardia and cardiac monitoring should be done while administration of neostigmine. Atropine ( $0.5 \mathrm{mg}$ i.v.) must be available if bradycardia occurs ${ }^{7,8}$. For those patients who fail pharmacological therapy the next step is to attempt endoscopic colonic decompression in conjunction with placement of a non-traumatic large diameter soft catheter rectal tube ${ }^{9}$. In this patient fortunately this step was not required.

\section{Conclusion}

ACPO is a potentially fatal condition, which can even occur after routine abdominal surgery. The diagnosis is one of exclusion and high clinical suspicion is necessary. When conservative measures fail neostigmine is the treatment of choice with high success rates.

\section{References}

1. Pereira P, Djeudji F, Leduc P, Fanget F, Barth X. Ogilvie's syndrome-acute colonic pseudoobstruction. Journal of Visceral Surgery 2015; 152: 99-105.

2. Vanek VW, Al-Salti M. Acute pseudo-obstruction of the colon (Ogilvie's syndrome). An analysis of 400 cases. Diseases of Colon Rectum. 1986; 29(3): 203-10.

3. De Giorgio R, Barbara G, Stanghellini V, et al. Review article: the pharmacological treatment of acute colonic pseudo-obstruction. Alimentary Pharmacology \& Therapeutics 2001; 15: 1717-27.

4. Chudzinski AP, Thomson EV, Ayscue JM, Acute colonic pseudo obstruction; Clinics in Colon and Rectal Surgery 2015; 28(2): 112-17.

5. Trevisani GT, Hyman NH, Church JM, Neostigmine: safe and effective treatment for acute colonic pseudo-obstruction, Diseases of Colon and Rectum. 2000; 43(5): 599-603.

6. Tangsucharit P, Takatori S, Sun P, et al. Do cholinergic nerves innervating rat mesenteric arteries regulate vascular tone? American Journal of Physiology Regulatory, Integrative and Comparative Physiology 2012; 303 (11): 147-56.

7. Ponec RJ, Saunders MD, Kimmey MBN. Neogstigmine for the treatment of acute colonic pseudo-obstruction. New England Journal of Medicine 1999; 341(3): 137-41.

8. Paran H, Silverberg D, Maya A, Shwartz I, Neufeld D, Freund U. Treatment of acute colonic pseudoobstruction with neostigmine. Journal of the American College of Surgeons 2000; 190(3): 315-18.

9. Harrison ME, Anderson MA, Appalaneni V, et al. The role of endoscopy in the management of patients with known and suspected colonic obstruction and pseudo-obstruction. Gastrointest Endosc. 2010; 71 (4): 669-79. 